\title{
Tangence
}

\section{Synthèse et postmodernité : de l'œuvre ouverte à l'œuvre fermée}

\section{Louis Hébert}

Numéro 39, mars 1993

La fiction postmoderne

URI : https://id.erudit.org/iderudit/025751ar

DOI : https://doi.org/10.7202/025751ar

Aller au sommaire du numéro

Éditeur(s)

Tangence

ISSN

0226-9554 (imprimé)

1710-0305 (numérique)

Découvrir la revue

Citer cet article

Hébert, L. (1993). Synthèse et postmodernité : de l'œuvre ouverte à l'œuvre

fermée. Tangence, (39), 37-50. https://doi.org/10.7202/025751ar d'utilisation que vous pouvez consulter en ligne.

https://apropos.erudit.org/fr/usagers/politique-dutilisation/ 


\section{Synthèse et postmodernité: de l'œuvre ouverte à l'œuvre fermée}

\section{Louis Hébert}

\section{Analyse pré-postmoderne 1}

La modernité a été (est?) une recherche de pureté dans chaque art, dira Clément Greenberg ${ }^{2}$. Chaque art a dû (doit?) s'autodéfinir selon les particularités de son médium. Chaque art rejettera tout dénominateur commun avec tout autre art. La peinture sera abstraite parce que la figuration relève aussi de la littérature, de la sculpture, etc.; elle atteindra alors son trait définitoire: la nature bidimensionnelle de son médium.

En transposant cette conception, on pourrait postuler que la littérature s'autodéfinit par la propriété exclusive qu'elle a de travailler - non pas le mot, qui appartient à tout discours verbal (théâtre, journalisme...), peut-être même pas le phonème, qui est du ressort de la littérature orale - mais la lettre.

\section{I.1. Génération des relations intégratives}

Dans cette démarche vers l'unité minimale, la littérature moderne fragmente une unité quelconque (du signifiant, du signifié, du référent (?)) en sous-unités: elle génère des relations intégratives entre les sous-unités et l'unité dont elles émanent et des relations distributionnelles entre les sous-unités ${ }^{3}$. L'évolution de la poésie illustrerait cette démarche d'autodéfinition. Pour la poésie traditionnelle l'unité minimale s'avère la proposition: le mot n'est pertinent que par son intégration dans cette unité supérieure. Puis l'unité minimale devient lexicale dans la poésie

1 J'approfondis ici des thèses avancées dans « Analyse et modernité [post-]. Fragments de Prochain épisode , à paraître en 1993 dans Protée.

2. Clément Greenberg, "La peinture moderniste*, Peinture, cabiers théoriques, $\mathrm{n}^{\infty}$ 8-9, p. 33-39.

3 Pour en savoir davantage sur les relations intégratives et distributionnelles, voir Problèmes de linguistique générale d'Émile Benveniste, Paris, Gallimard, 1966, p. 255 et s.). 
spatialiste et concrète, détruisant la nécessité d'une relation intégrative du mot. Enfin, la poésie lettriste ${ }^{4}$ éliminera la nécessité d'une relation intégrative de la lettre au sein du mot.

\section{I.2. Marquage des relations intégratives}

Or la fragmentation doit être marquée. Elle pourra l'être, semble-t-il, de deux façons: soit par traitement allomorphe des sous-unités, soit par élimination d'une des sous-unités (cette élimination constitue une opération quantitative faisant s'approcher la ou les parties restantes du statut du tout en diminuant le nombre de parties). J'appellerai la première technique allomorpbie distributionnelle et la seconde, élimination distributionnelle.

Ainsi, pour reprendre l'exemple de la poésie, la dissociation du vers traditionnel en sous-unités sera marquée, enregistrée, par la non-correspondance, par enjambement et rejet, entre coupe syntaxique et coupe du vers. Allomorphie distributionnelle.

L'apparition de la poésie lettriste et de la poésie phonétique illustre bien le deuxième mode de marquage analytique: la poésie traditionnelle, qui conjoignait des aspects visuels et sonores, se trouvent dissociée en deux domaines autonomes, poésie "visuelle" et poésie "sonore", qui peuvent expulser le codomaine. Élimination distributionnelle.

\subsection{Génération des micro-relations intégratives}

La démarche analytique se butera à deux limites, dont elle tirera un profit esthétique. Première limite. L'analyse s'effectuera

4 Le Groupe $\mu$ (*Avant-gardes et rhétoriques*, Les avant-gardes littéraires au $X X^{*}$ siècle, Budapest, Académiai Kiado, 1986) a proposé, pour classer certains genres poétiques, une grille à quatre variables groupées en deux catégories: contenu/expression; unité léxématique/unité infralexématique. Les poèmes traditionnels et spatialistes jouent sur le contenu avec comme unité de base le lexème; la poésie concrète a pour champ principal le niveau de l'expression avec comme unité le lexème; finalement, la poésie lettriste travaille sur le plan de l'expression avec des unités de base qui sont plus réduites, les graphèmes et traits graphiques distinctifs. Je diverge d'opinion quant à la poésie traditionnelle: les aspects de l'expression et du contenu du mot ne sont pas négligeables, mais ils sont subordonnés à leur inclusion dans une syntaxe propositive. 
sur une unité insécable (du signifiant, du signifié ou du référent (?)): on aura voulu générer des relations intégratives à partir d'une unité qui était déjà - en fait ou en apparence - l'unité minimale du parcours dissociatif.

Ainsi la fragmentation du domaine lettriste en traits graphiques même inévitablement au point (non pas le point signe de ponctuation), matrice graphique de toute lettre. En franchissant un certain seuil (sans doute situé avant le point), la démarche vers la spécificité retourne son arme contre elle et aboutit à l'enregistrement d'une "caractéristique partagée": le poème devient objet pictural. On passe du sens à la sensation, de l'abstraction sémantique à la pure concrétion sensuelle 5 . La modernité telle un gant retourné devient postmodernité, dans cette hybridation.

5 Le pun, fragmentation du signifiant suivie d'une concaténation avec d'autres fragments de signifiants, vise l'ouverture sémantique. Cependant, il existe probablement un seuil où l'ouverture par diminution de la redondance du formant atteint un champ de possibilités trop grand et est ressenti comme phénomène de concrétisation, de réification: dans cha, je peux lire chat, cbapon, cbaperon, etc.; mais si je ne maintiens qu'un $c$, passe-t-on de la polysémémie à une sorte de pansémie par défaut, par impossibilité d'embrasser le champ des possibles? Au delà d'un certain seuil, le grand nombre des possibles tue la lecture du possible. Un phénomène analogue se produit en peinture. Dans les œuvres de Picasso et de Braque, les parties d'objets fragmentés évoquent moult objets, mais passé un certain seuil, ils n'en n'évoqueront plus en particulier, en devenant eux-mêmes objets, et non plus représentation d'objets. La question se pose: la peinture *abstraite* représente-t-elle tout objet possédant tel trait pertinent qu'elle manifeste? tout objet? un objet étrange d'un monde possible: cet objet que représente telle œuvre de Riopelle? La poésie atteindra cette même abstraction-sens, au niveau cognitif, cette concrétion-sensation, au niveau pragmatique, par la fragmentation de son signifiant: en détruisant la signifiance sémantique (Benveniste), on plonge dans la seule signifiance sémiotique, seule mode de signifiance de la peinture, de la musique, etc. (pour J.-M. Léard (1981), la modernité (québécoise) en littérature apparaît dans une destruction progressive du niveau sémantique). D'ailleurs cet aboutissement n'est que l'exacerbation de sa nature même: l'accentuation de la fonction poétique se fait en quelque sorte au détriment de la fonction référentielle. La fonction transitive du langage (vers un pseudo-référent externe) est (au moins partiellement) déviée (et non détruite), au profit de sa fonction réflexive: l'accent ainsi mis sur le signifiant (et aussi le signifié) ne peut qu'augmenter la part des sens et mener à l'abstraction-concrétion. Ce qui était le signe de quelque chose (au moins en apparence) devient signe de lui-même, il avait toujours été signe de lui-même (tout signe est avant tout signe de lui-même), mais on ne le voyait que comme signe d'autre chose. 
40

Autre exemple. Dans Procbain épisode ${ }^{6}$, Hubert Aquin dissocie un acteur "biologique", unité réputée minimale eu égard aux contraintes de l'impression référentielle, pour en extraire les actants constitutifs: JE qui veut se suicider devient JE' et $\mathrm{H}$. de Heutz, l'agent ennemi que JE' doit abattre. Ou encore on dissocie le personnage de $\mathrm{K}$ en $\mathrm{K} 1$, amante de JE, et $\mathrm{K} 2$ amante de $\mathrm{H}$. de Heutz. Deux lectures sémiotiques opposées sont confrontées: celle qui dissout les relations distributionnelles par la fusion de deux unités de même niveau en une seule, par exemple l'unité $\mathrm{K} 1$ et l'unité K2 sont fusionnées en une unité de même niveau, $\mathrm{K}$ ' duplice; et celle qui établit une relation intégrative, par exemple entre les sous-unités $\mathrm{K} 1$ et $\mathrm{K} 2$ et l'unité intégrative $\mathrm{K}$, relation "aberrante" qui scinde un "corps" en deux. L'éthos de l'œuvre réside dans l'impossibilité de maintenir l'une ou l'autre de ces deux lectures tout au long du récit, alternance.

Deuxième tension créatrice. La conséquence ultime de la dissociation analytique de la modernité réside dans la confusion entre altérité et répétition de l'identique 7 . Le découpage d'un continuum donné (signifiant, signifié ou référent (?)), tel qu'il est effectué par un système de signes, doit respecter des règles pour éviter l'ambiguité. Ces règles, dont celles touchant la notion de marge de sécurité et de variante facultative, ont été définies par le modèle phonologique de Troubetzkoï. Certaines pratiques modernes les violent pour en tirer un effet esthétique. Ainsi la musique et la peinture micro-tonales fragmentent-elles le domaine d'un signe en plusieurs micro-domaines: le domaine de S1 (un demi-ton par exemple) est subdivisé en S1' + S2 + Sn (deux quarts de ton, par exemple).

Ces pratiques tendent à ce que l'altérité soit perçue comme répétition de l'identique en vertu de deux "risques". Le décodeur pourra ne pas posséder la compétence requise pour discriminer les micro-signes: soit qu'il ne les percevra pas, par "paresse sémiotique "ou par la limitation structurelle de l'appareil sensitif humain ${ }^{8}$; soit qu'il éliminera la différence perçue en recourant au

6 Pour des explications plus complètes, voir * Analyse et modernité [post-]. Fragments de Prochain épisode», op. cit.

7 J'extrapole ici à partir de propositions de Nicolas Ruwet (1972): certains signes de la musique moderne ne peuvent être perçus.

8 À la limite, de telles œuvres pourraient postuler un encodeur et un décodeur machine qui étendraient le champ des perceptions humaines 
principe de variante facultative d'un même signe (comme lorsqu'un locuteur français entend un $r$ uvulaire et un $r$ lingual). Les poétiques à micro-signe exigent donc une compétence de décodage accrue voire impossible, en définitive elles marquent ainsi l'altérité de l'œuvre en regard des instances de création et de réception. Allomorphie distributionnelle.

\section{Synthèse postmoderne}

Une fois arrivée au bout (?) de sa démarche de fragmentation, la modernité se renverse et devient postmodernité. Guy Scarpetta 9 prolongera la pensée de Clément Greenberg en postulant l'impureté de la postmodernité. L'ouvre postmoderne n'aurait plus pour objectif l'enregistrement des unités minimales propres à chaque discours, à la catégorie moderne/postmoderne, serait donc homologue la catégorie analyse/synthèse. Ainsi la postmodernité poserait, notamment, comme éléments (prétendument minimaux?) chacun des domaines que la modernité a analysés (analyse toujours?), danse, vidéo, sculpture, littérature, et procéderait à leur combinaison dans une unité intégrative, l'" installation ", "nom postmoderne de l'œuvre d'art "10.

Il semble qu'il y ait deux types principaux de synthèse qu'on pourrait appeler la restructuration et la méta-totalité. Dans la restructuration, les parties d'un objet analysé sont groupées de façon aléatoire: toutes les parties y sont, mais manque la cohésion systémique (qui est plus que la seule co-inclusion dans un tout de pression) présente avant l'analyse. Ainsi on supprimera la redondance "naturelle" qu'il y a, par exemple, entre main et avant-bras dans une toile cubiste. Cette pratique semble comme une synthèse après analyse formelle. Premier temps de la postmodernité. Deuxième cas: la synthèse de parties appartenant à des touts distincts, comme dans l'installation.

et maintiendraient la possibilité même d'existence de l'œuvre (un oscilloscope pourrait très bien discerner des micro-signes émis par un synthétiseur sonore, mais imperceptibles tant pour l'encodeur que le décodeur). On peut concevoir des œuvres pour appareils, des œuvres exclusivement pour telle espèce d'animaux ou d'insectes (en jouant avec des signes inaccessibles à l'appareil sensitif des autres espèces)!

9 Guy Scarpetta, L'impureté, Paris, Grasset, 1985.

10 René Payant, *Une ambiguitté résistante: l'installation *, Vedute, Montréal, Trois, p. 338. 
42

Évidemment une démarche synthétique ne saurait aboutir au même type d'objet que l'objet au point de départ d'une démarche analytique: la constitution de celui-ci est de l'ordre du donné, d'une forme de nature, d'un déjà-là (même si la recherche de la pureté semble viser à l'élimination des scories posées sur l'objet "naturel ": la "pure" poésie, la "pure " peinture, etc.); tandis que celui-là relève d'un construit, d'une culture, d'une hommerie.

La différence réside dans la relation entre chacune des parties entre elles (relations distributionnelles) et entre chacune des parties et le tout (relations intégratives). Dans le cas des objets soumis à l'entreprise moderne, les relations de partie à partie et de la partie au tout relèvent de la cohésion, de la pure fusion. Dans le cas de l'objet résultant de la synthèse postmoderne, la relation de partie à partie appartient à la juxtaposition. Mais tout se passe comme si, en vertu de sa nature même, le Tout surajoutait à cette hétérogénéité un liant de cohésion purement mécanique. Le postmoderne se sert de la fonction cohésive du tout de pression et l'oppose à la fonction juxtapositive des parties hétérogènes: "Ces éléments vont ensemble parce qu'ils sont ensemble ", affirme l'œuvre postmoderne.

Se pose donc la question d'une typologie des objets. La modernité interroge la partition: il semble y avoir des objets insécables, des unités minimales, à tout le moins dont la scission projette dans un autre domaine; la postmodernité questionne la fusibilité des parties au sein d'un objet: toutes les parties sont fusibles par simple co-présence dans un tout de pression, mais en même temps cette fusion, en réalité cette juxtaposition, doit être ressentie comme artificielle pour que l'œuvre obtienne son plein effet de culture. L'éthos de la partie postmoderne provient de ce que, telle une dilution homéopathique, elle conserve le souvenir d'un ensemble autre dont elle était une partie "naturelle". Ce souvenir s'oppose au lien construit contracté dans l'ensemble postmoderne, conflit temporel entre le "maintenant " homogénéisant et l' "avant "spécifiant. La postmodernité réside dans cette tension. Tout se passe comme si par le souvenir que chaque partie transporte du tout dont elle est extraite, l'œuvre postmoderne réussissait à former un tout non pas de parties mais de touts, de touts en parties...

La postmodernité est une pensée indicielle.

L'œuvre postmoderne est absence présentifiée: celle de cette partie qui fait que le tout est plus grand que la somme de ses 
parties, cette partie qui n'est peut-être autre chose que la cohésion du donné. Elle tente en quelque sorte de projeter la cohésion paradigmatique sur l'axe des concaténations, mais en marque l'impossibilité.

\section{De l'œuvre ouverte à l'œuvre fermée}

Eu égard à la catégorie analyse/synthèse, l'œuvre ouverte serait postmoderne, l'œuvre fermée, moderne. Toutes deux forment une catégorie graduelle: entre elles se placent deux états intermédiaires, l'œuvre semi-ouverte et l'œuvre semi-fermée, tandis que toutes s'opposent à l'œuvre neutre, métaterme pour désigner l'œuvre qui n'est ni ouverte ni fermée. Cette typologie repose sur deux variables oppositives: sens littéral/sens figuré. .Chacune de ces variables s'inscrit dans une opposition privative: présence ou absence de sens littéral, présence ou absence de sens figuré. Dans l'éventualité ou le sens figuré est présent, la relation jouera alors aussi sur une opposition graduelle: sens figuré unique/multiple. Finalement, pourra servir de critère la modalité d'existence: virtuelle/actuelle/réalisée (trichotomie décomposable en multiples oppositions binaires).

Globalement, la réflexion sémiotique en jeu dans les différents degrés d'ouverture et de fermeture est moderne: on élimine le degré figuré (élimination distributionnelle) ou on génère l'allomorphie (allomorphie distributionnelle) par la disproportion entre l'étendue du sens littéral en regard du sens figuré. Ce qui n'empêche pas de considérer l'œuvre ouverte comme postmoderne (d'ailleurs l'impureté de l'œuvre postmoderne proviendraitelle de la co-présence du moderne et du postmoderne?). Car la catégorie moderne/postmoderne est, par définition, relative aux termes mis en présence: un même terme peut à la fois être moderne par rapport à un terme et postmoderne par rapport à un autre ${ }^{11}$.

11 Il en va ici comme de l'opposition nature/culture: dans la tripartition bien connue des espaces du roman québécois traditionnel (entendre pré-moderne), on oppose à la fois la forêt à la terre (au sens de terre cultivée) et la terre à la ville, le terme de aterre. occupe donc successivement la position de culture par rapport à la forêt et de nature par rapport à la ville. 
44

\section{III.1. L'œuvre neutre}

Parler d'œuvre neutre pour classer le discours communément appelé "non littéraire" implique un certain regard: celui d'une non-séparation fondamentale. L'œuvre neutre et les autres types d'œuvre appartiennent à l'articulation d'une même catégorie sémantique. Simplement, l'œuvre neutre nie et le sens littéral et le sens figuré. Il y a LE sens. La polysémémie (qu'amène la coprésence du sens littéral et du sens figuré), inévitablement présente dans les structures virtuelles de tout discours, ne sera pas actualisée. Toute double isotopie tend à être entravée par des opérations de surdétermination de l'isotopie actualisée et sousdétermination des isotopies virtualisées. De plus, l'énoncé construit un narrataire "coopératif ", qui lui évite d'avoir incessamment à surdéterminer et sous-déterminer les diverses isotopies.

\section{III.2. L'œuvre semi-ouverte}

-Une théorie du sens multiple, nous dit le Groupe $\mu$, suppose que soit d'abord formalisée une théorie du double sens, laquelle suffit du reste à rendre compte d'une bonne part des faits de littérature "12. Le double sens (simple, et non multiple) fonde les œuvres semi-ouvertes, la littérature "traditionnelle" (entendre prémoderne). "Le Vaisseau d'or" de Nelligan (vaisseau = poète) et "L'albatros" de Baudelaire (albatros = poète) constituent des modèles du genre ${ }^{13}$.

La métaphore, type même du procédé de double sens, est un connecteur isotopique entre le sens littéral et le sens figuré. Mais ces deux sens ne sont pas en relation symétrique, il y a une rection allant du sens littéral vers le sens figuré. Le sens littéral est le signifiant du sens figuré. Le rapport forme sensible/forme de sens entre signifiant et signifié se trouve comme transposé dans le

12 Groupe $\mu$, Rhétorique générale, Paris, Seuil, 1982, p. 38.

13 Cette superposition paradigmatique générée par le connecteur isotopique sera souvent déployée en successivité syntagmatique biisotopique. Dans " Le Vaisseau d'or . de Nelligan (on pourrait aussi bien parler de "L'Albatros " de Baudelaire), nous passons de l'incipit biisotopique: Ce fut un grand Vaisseau à l'excipit, où deux termes mono-isotopiques (en regard des isotopies "navire" et "poète *) sont syntaxiquement donnés comme équivalents et contractent ainsi un statut bi-isotopique: *Qu'est devenu mon cœur, navire déserté?» Cette biisotopie confirme celle du lexème * Vaisseau • de l'incipit. 
seul contenu: le sens littéral joue un rôle homologue à celui du signifiant, par son orientation syntaxique première dans la génération de la signification (on passe du signifiant au signifié, du littéral au figuré) et sa fonction de représentation (figurer quelque chose d'autre).

\section{III.3. L'œuvre ouverte}

Le processus de synthèse peut être repéré dans une pratique comme celle de l'œuvre ouverte. Dans ce type d'œuvre, en effet, un signifiant condense plusieurs signifiés. Kafka, nous dit Eco, représente le type même de l'œuvre ouverte: "procès, château, attente, condamnation, maladie, métamorphose, torture ne doivent pas être pris dans leur sens littéral."14. "Ne doivent pas être pris qu'au sens littéral ", devrait-on dire: Robbe-Grillet s'insurgera contre les "prétendus descendants " de Kafka qui "ne faisaient que reproduire le contenu métaphysique et oubliaient le réalisme du maître " 15 .

Ainsi conçue, l'œuvre ouverte ne serait pas de nature qualitativement différente de l'œuvre traditionnelle, elle ne ferait qu'exacerber la tendance à la polysémémie avec pour limite la pansémie: ce moment où une ouvre, voire un signe, engloberait l'univers de la signification. L' "univers" joycien rivalise avec le pansémisme de l'Univers. La partie devient le Tout et s'englobe elle-même comme partie. Mais l'œuvre qui en un mot exprimerait le Tout ne serait pas la diagrammatisation, la représentation d'ellemême, puisque la multiplicité de sa contenance serait sentie tensivement avec le minimalisme du formant. Elle serait une partietout. Voilà qui explique l'affinité plus grande entre baroquisme et polysémémie et le lien plus grand entre minimalisme et œuvre fermée: il semble en effet possible de corréler des catégories telles classique/baroque (H. Wölfflin) et Abstraction/Einfüblung ${ }^{16}$ (W. Worringer) à celle de modernité / postmodernité.

14 Umberto Eco, L'ceuvre ouverte, Paris, Seuil, 1965, p. 22.

15 Alain Robbe-Grillet, Pour un nouveau roman, Paris, Gallimard, 1963, p. 180.

16 Wilhelm Worringer (Abstraction et Einfüblung. Contribution à la psycbologie du style, Paris, Klincksieck, 1986) segmente les productions artistiques en deux classes, celles qui relèvent de l'Abstraction et celles qui relèvent de l'Einfüblung. Chacune des pratiques implique des paradigmes opposés de caractéristiques picturales. Einfüblung et Abstraction 
46

Dans l'cuvre ouverte, le sens littéral n'occupe que la portion congrue de la signification totale. L'œuvre ouverte tend en quelque sorte à éliminer le sens littéral (démarche impossible) en l'enterrant sous le nombre des sens figurés. En fait, elle se trouve à doter d'une structure allomorphe le sens littéral, unique, et le sens figuré, multiple (se rappeler qu'il y a du moderne dans le postmoderne (et l'inverse?)). Par cette disproportion, le rapport sens littéral/sens figuré devient homologue à celui entre le signifié et le référent: le signifié ne recèle que les traits essentiels au départage d'avec les autres signifiés: "bien des propriétés du référent n'ont 'pas de place dans le signifié, car elles n'interviennent pas dans les classifications inhérentes à la langue "17. Se trouve donc mise en abyme, dans la seule dimension du signifié, la disproportion du sens des langues naturelles en regard du sens du monde naturel. L'œuvre modélise l'univers.

Il semble possible de distinguer deux techniques principales de production d'ouverture, qui constituent aussi deux temps dans l'apparition de l'œuvre ouverte. D'abord, on exacerbera le caractère minimalement bi-isotopique du langage poétique: dans l'œuvre de Kafka, le lexème "château " représente ce château (sens littéral), mais aussi la centralisation bureaucratique, le domaine de Dieu, le domaine du père, etc. Le travail s'opère dans la dimension du signifié.

L'image surréaliste, conciliant deux termes éloignês, ouvre la voie au pansémisme: un mot pouvant signifier tout, donc chaque partie du Tout ${ }^{18}$. Ne reste plus qu'à signifier tout - en même

s'opposent par les catégories suivantes: individuel/collectif, naturalisme/style, monde organique/monde inorganique, espace tridimensionnel/plan surface, arrondi/ligne droite, apparition optique/ apparition tactile. La Grèce forme un exemple du premier type et l'Egypte, du second, tandis que Byzance se trouve à mi-chemin. Or le choix d'une pratique ou de l'autre, nous dit Worringer, qu'il soit individuel ou sociétal, relèverait du rapport au monde. Privilégier les œuvres de l'Einfüblung, de la postmodernité, serait symptomatique d'un rapport non anxiogène à l'univers et vice versa.

17 Oswald Ducrot et Tzvetan Todorov, Dictionnaire encyclopédique des sciences du langage, Paris, Seuil 1972, p. 317-318.

18 Le surréalisme a compris que la métaphore étend l'identité de l'intersection, entre deux touts en présence, à toutes les parties de ces deux touts, formant un nouveau tout par synthèse, où altérité et identité sont en tension. Autrement dit, on construit, a posteriori, des relations intégratives qui justifieraient le rapprochement métaphorique. La métaphore 
temps. Ce que fera le pun joycien: deux, trois, dix racines différentes s'enchevêtrent dans un même formant. L'impureté du signifié ouvert, constitué d'une superposition de signifiés, se trouve corrélée au "monstre" (la postmodernité est amour du monstre) que devient le signifiant: mise en abyme. Le tout de pression est ici le formant. La fracture fondamentale entre le langage et le "réel" est diagrammatisée, cette tension entre la diversité référentielle et sa réduction par la langue chargée de la représenter ("arbre" subsume une foule d'individus)... Mais cette réduction fondamentale du langage, qui procède par analyse sémique et obtient ainsi une justification "logique", s'oppose alors au pun, juxtaposition soit aléatoire, soit structurée par affinité de signifiants.

Le choix paradigmatique d'un seul formant par position syntagmatique, rendu nécessaire par la linéarité du discours, se trouve comme nié par la stratification du formant du pun. Cette superposition paradigmatique (celle du formant comme celle du signifié), niant la linéarité du discours (encore plus que dans l'ouvre semi-ouverte), laquelle est liée au temps, appelle une aperception spatiale.

La première technique se limite à l'allomorphie distributionnelle (cette différence quantitative entre sens littéral et sens figuré), tandis que la seconde utilise, en plus, l'élimination distributionnelle en ne conservant qu'une partie des différents formants constitutifs du formant pun. Mais leur impact est postmoderne. Enfin, les deux techniques d'ouverture peuvent évidemment être combinées par un pun dont une ou plusieurs racines, telles des missiles à têtes multiples, libéreront chacun leur charge de condensation de signifiés obtenue par la première technique.

\section{III.4. L'œuvre semi-fermée}

Dans l'œuvre semi-fermée, le double sens est toujours présent, mais entravé, nié par l'œuvre. Ainsi, dans L'écume des jours, Boris Vian s'amuse-t-il sérieusement à détruire le transfert

ne note que les rapports qualitatifs différence/ressemblance, mais ignore (ou feint d'ignorer) les données quantitatives (le partage d'un trait commun n'élimine pas la masse des traits différentiels). Les surréalistes n'ont fait que mettre l'accent sur l'autre versant de la métaphore, l'altérité. Si la métaphore classique est un outil postmoderne par sa fonction synthétique, c'est dans son utilisation surréaliste qu'elle deviendra un tout de pression ressenti comme *arbitraire *, donc encore plus postmoderne. 
métaphorique. Lorsqu'on nous dit qu'un personnage est "planté " là, on ajoute qu'il prend racine! Il y a un équilibre quantitatif entre sens littéral et sens figuré, comme dans l'œuvre semiouverte, mais l'équilibre manifeste une rection qui ramène au sens littéral. Coexistence de deux lectures paradigmatiques actualisées par le narrataire dont l'une seulement sera réalisée: le texte "prévoit" ces deux lectures et tire de leur confrontation un éthos particulier fait d'humour et de pensée déréelle.

Breton fournira un deuxième exemple. Se moquant de l'analyse traditionnelle cherchant à tout prix un sens figuré: "Lendemain de chenille en tenue de bal veut dire: papillon. Mamelle de cristal veut dire: une carafe, etc. Non, Monsieur, ne veut pas dire: rentrez votre papillon dans votre carafe. Ce que Saint Pol-Roux a voulu dire, soyez certain qu'il l'a dit "19. Dans l'œuvre semi-fermée, il faut résister à la tentation de passer du sens littéral au sens figuré, et prendre au mot (ou au pied de la lettre) la métaphore, si je puis dire. Breton marque un pas de plus vers l'œuvre fermée en ce qu'il dénonce la possibilité même de résonner contre le sens littéral. Cependant, peut-on dire que l'œuvre surréaliste, structurellement, n'appelle pas le faire-valoir, la présence parasitaire du sens figuré (surtout dans le contexte de production, où les lecteurs (surréalistes ou non) possédaient un appareil interprétatif formé à l'œuvre semi-ouverte)?

\section{III.5. L'œuvre fermée}

Barthes a parlé d'œuvre fermée. La Nouvelle critique aurait eu pour fonction de transformer en sens pluriel le sens singulier que prêtait à l'œeuvre l'Ancienne critique, de transformer ainsi "l'œuvre fermée en œuvre ouverte" 20 . Entendue ainsi, la fermeture ne relève pas de l'œuvre (toujours ouverte, à divers degrés), elle est une myopie du critique.

Pourtant, certains textes, paradoxalement proches de la Nouvelle critique, appellent une forme de fermeture interprétative et inscrivent cette visée dans leur structure: nous pensons aux textes d'Alain Robbe-Grillet, "Le mannequin" ${ }^{21}$ en particulier.

19 André Breton, Point du jour, Paris, Gallimard, coll. * Idées *, 1937, p. 27.

20 Roland Barthes, Critique et vérité, Paris, Seuil, 1966, p. 50.

21 Alain Robbe-Grillet, Le mannequin *, Instantanés, Paris, Minuit, 1962, p. 9-13. 
Dans cette œuvre fermée (au sens maintenant restreint du terme) le mode d'existence sémiotique du sens figuré n'est plus de l'ordre de la réalisation, comme dans l'œuvre semi-ouverte et ouverte, ni de celui de l'actualisation, comme dans l'oeuvre semifermée, il découle simplement de la virtualité.

Ce type d'œuvre va volontairement s'expurger (en bonne partie du moins) des procédés de double sens, notamment la métaphore et la comparaison (projection syntagmatique de la métaphore). Il pourra même, imitant en cela l'œuvre semi-fermée, se gausser à l'avance (et donc indiquer comme non avenue) de toute vélléité de recours à un sens figuré, le sens littéral est invinciblement tourné sur lui-même: "L'anse [de la cafetière] a, si l'on veut, la forme d'une oreille, ou plutôt de l'ourlet extérieur d'une oreille; mais ce serait une oreille mal faite, trop arrondie et sans lobe, qui aurait ainsi la forme d'une "anse de pot" "22.

Une telle littéralité n'empêche aucun lecteur de tenter d'exploiter les structures virtuelles pour y déloger un quelconque sens figuré, mais il le fera au corps défendant et de la poétique de l'œuvre et de sa structure, pure distorsion (ne peut-on "distorsionner" les ouvres, les saturer comme une guitare beavy metal?): rien n'empêche de lire un conte érotique dans l'annuaire téléphonique. Après tout, "Rien n'est plus ouvert qu'un texte fermé. Mais son ouverture est l'effet d'une initiative extérieure, une façon d'utiliser le texte et non pas d'être utilisé par lui, en douceur"23. La béance laissée par l'absence de sens figuré peut donc susciter, comme le note Barthes ${ }^{24}$ a propos de l'état zéro" de l'anecdote dans Le voyeur, "toutes sortes d'investissements métaphysiques" intempestifs, ou, ce qui revient au même, un appel à l'absurde. Toutes échappatoires dénoncées par RobbeGrillet, qui applique le même principe à l'écriture de ses œuvres qu'à la lecture de l'univers: "le monde n'est ni signifiant ni absurde. Il est, tout simplement "25.

Si dans la métaphore surréaliste, l'altérité la plus lointaine est contrainte, par pression du trope, à l'identité, à l'opposé, dans

22 Ibid., p. 10.

23 Umberto Eco, "Textes "fermés" et textes "Ouverts", Lector in fabula, Paris, Grasset, 1985 , p. 71.

24 Roland Barthes, "Littêrature littérale*, Essais critiques, Paris, Seuil, 1964, p. 69 .

25 Alain Robbe-Grillet, Pour un nouveau roman, op. cit., p. 21. 
50

l'ouvre fermée, le signe n'est signe que de lui-même (tout signe est, avant toute transitivité, signe de lui-même). Ce qui ne veut pas dire que le texte ne tolère aucun investissement de sens figuré, mais les possibilités de cet investissement sont structurellement réduites (encore plus que dans l'ouvre semi-fermée surréaliste) et poétiquement déclarées non valides par le producteur. Mais, ici encore, une partie de l'êthos ne réside-t-elle pas dans la confrontation entre le texte et un lecteur "incompétent"? $\grave{A}$ cet égard se trouvent sans doute des marques fines d'un tel narrataire investissant "métaphysiquement " le texte.

L'œuvre fermée est donc le résultat d'une démarche de fragmentation par élimination distributionnelle (le procédé le plus extrême de la modernité) qui vise à démontrer l'autonomie du sens littéral. Celui-ci, par la disparition du sens figuré des structures actuelles et réalisées, progresse vers le statut du tout, devient le tout, occupant l'entièreté du sémantique. CEuvre moderne. Pourtant comme le mentionne Guy Scarpetta, "le roman est, par nature, un art impur ": il a "avalé " le théâtre (le dialogue), la poésie (la prosodie de Flaubert, le pun joycien, etc.), l'essai (Dostoïevski, Musil, Broch), etc. ${ }^{26}$. Ce serait donc dans cet espace restreint (devenir le plus pur possible malgré l'impureté fondatrice) que se meut l'œuvre fermée narrative: sans personnage, sans action, sans sens figuré. Pour certains, ce n'est plus du roman, de la nouvelle, on aurait dissocié l'unité minimale. Pour d'autres c'est là que réside la propriété de la narrativité: la description. D'ailleurs, le passage de l'œuvre ouverte à l'œuvre fermée implique, pour la critique, le passage en quelque sorte de l'interprétation à la description de l'œuvre, dont on sait la nature analytique. Chaque forme de critique semble susciter un type d'œuvre adapté à la grille qu'elle utilise (ou est-ce l'inverse?): comme la découverte de la psychanalyse a produit des romans inscrivant des marques pour un décodage psychanalytique, l'œuvre fermée est le type même de l'œuvre sacrifiée à l'uavancement de la science "structuraliste.

\section{Conclusion disparue par élimination distributionnelle}

